\title{
Evaluación de la riqueza y composición de macrohongos en términos de distribución temporal en tres biotopos de la finca Santa Maura - Estación Biológica Juan Roberto Zarruck, reserva natural Datanlí-El Diablo
}

\section{Iris Lilliam Saldivar Gómez}

\section{Recibido: agosto de 2015 / aceptado: octubre de 2015}

Los hongos en Nicaragua son un grupo en el que hasta el momento se reportan 184 especies macrohongos (González \& Orozco, 2007). Líquenes se reportan 275 especies (Breuss, 2003) y 194 especies de hongos hyphomycetes (Delgado, 2011). Estas son cifras que se encuentran en constante aumento, sujetas al esfuerzo muestral. En la Estación Biológica Juan Roberto Zarruck se reportan 73 especies. La presente investigación forma parte de un proyecto planeado para cinco años de investigación, donde se analiza la composición espacial y temporal de las especies de macrohongos encontrados en tres biotopos ubicados en parches de bosques de la Estación Biológica Juan Roberto Zarruck, durante los años 2013 y 2014. La composición de especies en cuanto a su riqueza y abundancia mostró variaciones en el año 2013 con respecto al 2014, siendo la riqueza de 52 especies reportadas en el 2013 y 35 especies en el 2014. También varió la abundancia de especies de 1068 individuos en el 2013 a 448 individuos en el 2014. El índice de riqueza de Shannon en ambos años no mostró un valor óptimo. La disminución en el número de individuos en 2014 y 2013 podría estar sujeta a las precipitaciones en la zona. El 57\% de especies de macrohongos en el 2013 y el 47\% en el 2014 obedecen a hábitos saprofitos, lo que indica que son principalmente descomponedores de madera en descomposición, siendo este dato valioso para entender su función en este ecosistema de bosque y los posibles efectos del cambio climático en esta taxa.

Palabras clave: Macrohongos / biotopos / abundancia / riqueza / saprofitos / cambio climático

Estación Biológica Juan Roberto Zarruck, Universidad Centraomericana. Correo electrónico: irisgg81@googlemail.com. 


\section{Introducción}

El reino fungí cuenta con aproximadamente 80000 especies clasificadas que poseen las más diversas formas. En Nicaragua han sido reportadas 184 especies de macrohongos (González \& Orozco, 2007). De líquenes se reportan 275 especies (Breuss, 2003) y 194 especies de hongos hyphomycetes (Delgado, 2011).

A nivel de Centroamérica este grupo no ha sido tan extensamente estudiado como las plantas vasculares. Sin embargo, en países como Costa Rica se han reportado 2000 especies en sus últimos inventarios y se estima que la riqueza de especies en dicho país podría llegar a ser de 20, 000 o 40000 (Mata, 2003). Las investigaciones fúngicas se encuentran en continuo aumento debido no sólo al interés ecológico de estos organismos en los procesos de reciclaje del bosque, sino también por el aumento de su interés como recurso económico.

El departamento de Jinotega está formado por importantes bosques premontanos con una vegetación caracterizada por roble encino, que tienen una marcada influencia altitudinal. Además, estos bosques albergan vegetación intrínsecamente ligada a niveles altos de humedad, con un marcado poder de regeneración ante las perturbaciones antropógenicas (Stevens, 1995), y corredores biológicos conectados con algunas de las áreas de conservación que forman parte de la reserva de biosfera Bosawas (MARENA \& FUNDAR, 2012).

Las nebliselvas facilitan el desarrollo de especies de hongos debido al alto contenido de materia orgánica que poseen y a su humedad por encima del 90\%. Es por ello que los hongos son excelentes para encontrar alteraciones en los parámetros ambientales de los bosques, ya que estos organismos son fuertemente influenciados por la temperatura, las variaciones en las precipitaciones anuales y la humedad (Kauserud et al., 2008).

Los factores que inciden o limitan la distribución de los macrohongos han sido poco o nada estudiados debido a la temporalidad de los mismos. Sin embargo se sabe que uno de los principales parámetros que inciden en la distribución de estos organismos es la humedad (CESEFOR Gestión Forestal Sostenible, 2013). Por ello, esta es la primera iniciativa a nivel Centroamericano que analiza la distribución y composición fúngica en términos espacio- temporales en un bosque nuboso.

Una línea base de especies obtenida en el año 2012 permitió tener el conocimiento de las especies presentes en el área, por lo que el presente estudio pretende evaluar el comportamiento de la riqueza y composición de los macrohongos presentes en tres biotopos de la Finca Santa Maura-Estación Biológica Roberto Zarruck, conociendo su distribución espacial y temporal en los años 2013-2014.

\section{Metodología}

\section{1. Área de estudio}

La Estación Biológica Roberto Zarruck está geográficamente ubicada en el área de conservación de la Reserva Natural Datanlí- El Diablo. Esta reserva comprende un área de 5,848 ha, conformadas por diferentes fincas privadas y áreas de 
investigación que se encuentran localizadas en el centro de la reserva y en su zona de amortiguamiento.

La estación biológica está ubicada en la finca Santa Maura, la cual tiene una extensión de 700 ha de terreno, utilizado en su mayor parte para el cultivo de Coffea arabiga. Esta finca tiene aproximadamente unas 300 ha de bosque tipo premontano con abundancia de Quercus y especies asociadas a la altura.

La Reserva Datanlí se caracteriza por ser un bosque de nebliselva con precipitaciones intensas durante nueve meses del año. El promedio de precipitaciones de la zona oscila entre los 2,400 $\mathrm{mm}$ a los 3,200 $\mathrm{mm}$ anuales (datos propios de la estación). La temperatura en la zona varía según la fisiografía, por lo cual estos cambios en el relieve provocan temperaturas promedio entre los 22.5 a los $20.5 \mathrm{C}^{\circ}$ anuales, según la altura de los diferentes biotopos.

El bosque está clasificado en esta área como bosque húmedo subtropical (bah-s) y bosque muy húmedo subtropical (bah-S). La composición del bosque en esta reserva se caracteriza por parches de bosque en diferentes estados de conservación. El uso de la tierra, principalmente en la zona de amortiguamiento, está compuesto por la siembra de café de sombra en algunas parcelas.

\subsection{Ubicación de los sitios de muestreo}

El muestreo de macrohongos se efectuó con la ubicación de 10 parcelas permanentes de $100 \mathrm{~m}^{2}$ (10 me $\times 10 \mathrm{~m}$ ). Las parcelas fueron ubicadas en los puntos de muestreo que representan diferentes biotopos. Estos biotopos presentan pequeñas diferencias en la composición de la vegetación, por lo que fueron caracterizados como Tacotal, Ripario y Bosque de Roble.

En cada parcela de $100 \mathrm{~m}^{2}$ se ubicó una pseudoréplica con cuatro parcelas de $2 \mathrm{~m}^{2}$ ubicadas en las esquinas de la parcela mayor y una sub parcela de $5 \mathrm{~m}^{2}$ ubicada en la parte central de la parcela de $100 \mathrm{~m}^{2}$. Estas pseudoréplicas permitieron optimizar el barrido de especímenes dentro de la parcela principal y al mismo tiempo proporcionaron datos más precisos de la presencia y ausencia de especies.

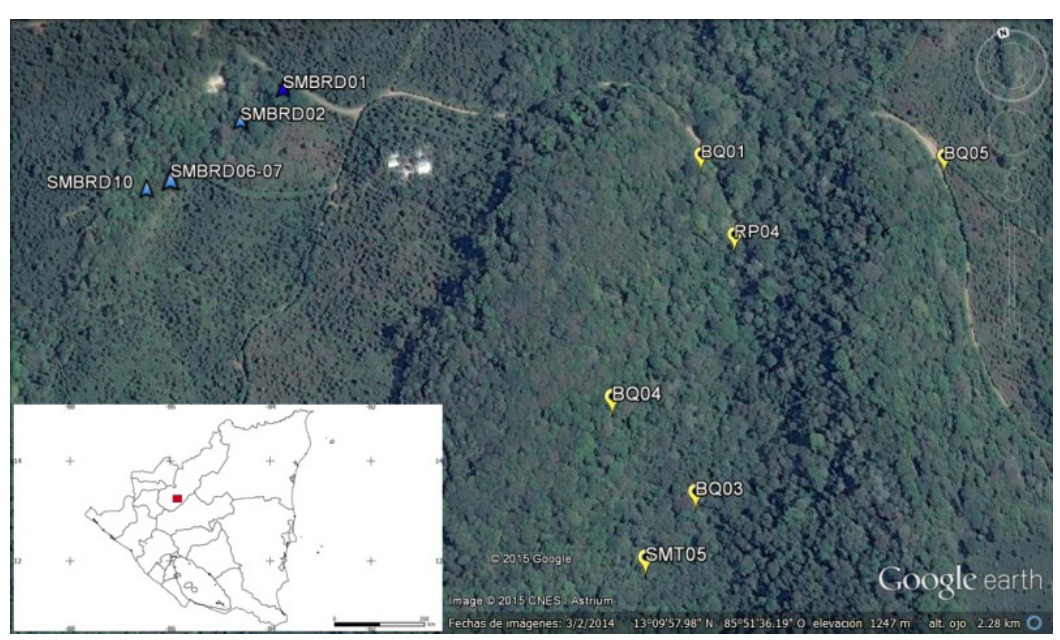

Ilustración 1. Ubicación de las parcelas en diferentes biotopos de Santa Maura 


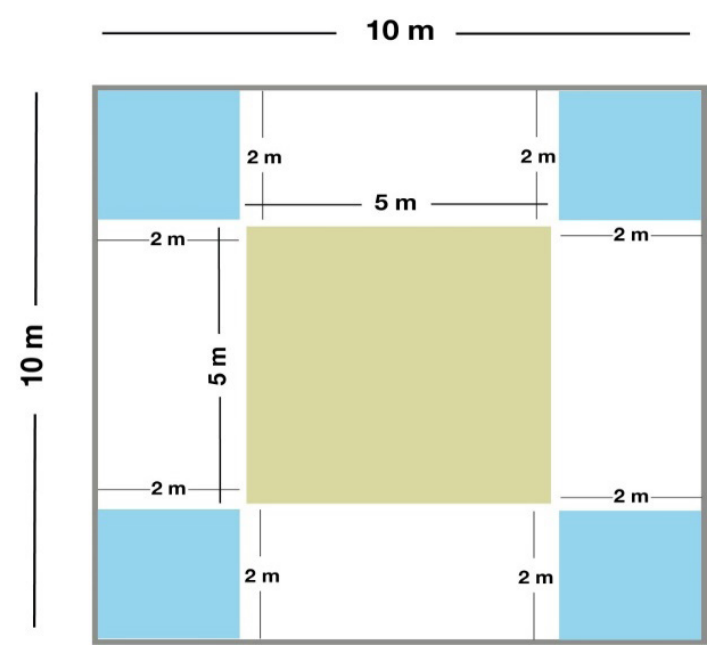

Ilustración 2. Estructura de las parcelas y las sub parcelas de muestreo

\subsection{Colecta del material biológico}

La recolecta de datos se efectuó en los meses de octubre 2013 y octubre 2014, posterior a los días más intensos de precipitaciones en esta zona. El número de parcelas que se establecieron corresponden al 5\% del total del área de bosque de la estación biológica Juan Roberto Zarruck, en la finca Santa Maura.

Se tomó como referencia para la colecta el listado de macrohongos de la estación biológica realizado en octubre 2012. Los especímenes colectados fueron debidamente descritos según las claves para macrohongos descritas en Agaric flora of the lesser Antilles (Pegler, 1983) y Micología (Müller \& Loeffler, 1976), Mycokey (Petersen \& Læssøe, 2014) y MushroomExpert (2015). Después de ello fueron envueltos en papel parafinado y trasportados en canastas para no dañar sus estructuras. Estos especímenes fueron llevados a laboratorio en donde se observaron sus esporas, tramas e hifas con la ayuda de un microscopio compuesto, con un aumento de 1000 x. Para las observaciones se utilizó el reactivo Hidróxido de Potasio al 10\% y reactivo de Melzer.

Las parcelas fueron debidamente georeferenciadas con un GPS de marca Garmin, modelo GPSmap 60csx.

\subsection{Análisis de la información}

Para el análisis de la información se utilizó el software R Project 3.1.2 con ayuda de los paquetes ROCR y Vegan.

Se realizó un análisis de la diversidad de especies en base a Shannon y su inverso, lo que se graficó en función de los biotopos muestreados. Se hicieron análisis de similitud de las especies en base a Clúster con la fórmula average. Se hizo un análisis de la diversidad de especies según tipo de bosque, donde los resultados se analizaron en base al índice de Shannon (Shannon \& Weaver, 1948). Se hicieron pruebas de rarefacción para observar la posible tendencia de encontrar nuevas especies en las áreas muéstrales. 


\section{Resultados}

Los estudios efectuados en los años 2012, 2013 y 2014 en los parches de bosque que corresponden a la Estación biológica Roberto Zarruck han permitido identificar 73 especies de macrohongos, los cuales incluyen diversas taxas del Reino Fungí.

Los análisis que se desarrollan a continuación son el resultado de dos años de esfuerzo muestral en diez parcelas permanentes ubicadas en parches de bosque destinados a áreas de conservación en la Estación Biológica Roberto Zarruck, finca Santa Maura-UCA.

Las parcelas muestreadas en el mes de octubre del año 2013 presentaron una riqueza de 52 especies, las cuales fueron encontradas en una abundancia de 1068 individuos y aproximadamente una densidad de 1.68 especies por $\mathrm{m}^{2}$ distribuidas en las 0.1 ha muestreadas. Durante el muestreo realizado en el año 2014 se encontraron 35 especies de hongos con una abundancia total de 448 individuos, lo que representó una densidad de cobertura de 0.44 individuos por $\mathrm{m}^{2}$.

En el año 2013, de las 52 especies de macrohongos encontradas, 21 especímenes fueron nuevos reportes para la Estación Biológica. Durante el muestreo de 2014 hubo nueve nuevos reportes de especies para la zona. Los siguientes macrohongos no se han reportado en otras zonas: Helvella atra, Campanella alba, Crinipellis scabella, Camillea sagrana, Mycena renati, Pluteus nanus, Engleromyces goetzei, Gymnopus iocephalus, Marasmius berteroi, Marasmius quercophilus, Mycena chusqueophila, Calvatia rugosa, Psathyrella conopilus, Marasmius tageticolor.

En el caso de los reportes nuevos, específicamente para el área comprendida en la Estación Biológica, fueron los siguientes tomando en cuenta los años de muestreo 2013 y 2014. Ver cuadros 1 y 2 :

Cuadro 1. Especies nuevas Estación Biológica Roberto Zarruck - 2013

\begin{tabular}{|l|l|}
\hline Amanita brunnescens & Mycena sanguinolenta \\
\hline Camillea sagrana & Naucoria striatula \\
\hline Campanella alba & Phellinus giluus \\
\hline Crinipellis procera & Pluteus leoninus \\
\hline Crinipellis scabella & Pluteus salicinus \\
\hline Crinipellis stipitaria & Podostroma alutaceum \\
\hline Entoloma sp & Ramaria stricta \\
\hline Gymnopillus sp & Ramaria zippelii \\
\hline Helvella atrata & Tricholoma myomyces \\
\hline Hohenbuelia geogenia & Tetrapyrgos nigripes \\
\hline Marasmius quercophilus & \\
\hline
\end{tabular}


Cuadro 2. Especies nuevas Estación Biológica Roberto Zarruck - 2014

\begin{tabular}{|l|l|}
\hline Mycena renati & Mycena chusqueophyla \\
\hline Pluteus nanus & Calvatia rugosa \\
\hline Engleromyces goetzei & Marasmius taeticolor \\
\hline Gymnopus iocephalus & Psathyrella conopilus \\
\hline Marasmius berteroi & \\
\hline
\end{tabular}

Todas las especies encontradas mostraron una abundancia absoluta por debajo del $15 \%$ de cobertura del terreno en el año 2013. A pesar de ello, se tomó como referencia las 14 especies con abundancias superiores a 10 individuos en todo el terreno. Las especies aquí señaladas con mayor número de individuos son especies que no requieren especificidad de hábitat y que muestran ciertos rangos de tolerancia a las variaciones de humedad. A continuación observaremos las tablas de especies comunes para los años 2013 y 2014:

Cuadro 3. Especies más abundantes según el tipo de bosque y el total de los individuos - 2013

\begin{tabular}{|l|r|r|r|r|r|}
\hline Especies & Tacotal & Ripario & Roble & Total & \multicolumn{1}{c|}{$\%$} \\
\hline Campanella alba & & & & & $\mathrm{n}=1068$ \\
\hline Coprinus disseminates & 52 & 103 & 26 & 181 & 17 \\
\hline Crinipellis procera & 51 & 0 & 4 & 55 & 5 \\
\hline Crinipellis stipitaria & 36 & 0 & & 39 & 3.6 \\
\hline Cyathus striatus & 10 & 1 & 20 & 31 & 3 \\
\hline Dacryopinax spathularia & 9 & 18 & 5 & 32 & 3 \\
\hline Marasmius cladophyllus & 24 & 0 & 0 & 24 & 2 \\
\hline Marasmius leprieurii & 17 & 0 & 10 & 27 & 2.5 \\
\hline Marasmius sp & 3 & 1 & 18 & 22 & 2 \\
\hline Marasmiellus volvatus & 21 & 0 & 41 & 62 & 6 \\
\hline Mycena sanguinolenta & 59 & 30 & 17 & 106 & 10 \\
\hline Scutellinia scutellata & 25 & 3 & 12 & 40 & 3.7 \\
\hline Ramaria stricta & 4 & 15 & 9 & 28 & 2.6 \\
\hline Xylaria polymorpha & 0 & 32 & 17 & 49 & 4.5 \\
\hline
\end{tabular}


Cuadro 4. Especies más abundantes según el tipo de bosque y el total de los individuos - 2014

\begin{tabular}{|l|r|r|r|r|r|}
\hline Especies & Tacotal & Ripario & Roble & Total & \multicolumn{2}{l|}{ \% } \\
\hline & & & & & $\mathrm{n}=448$ \\
\hline Calvatia rugosa & 0 & 0 & 60 & 60 & 13 \\
\hline Camillea sagrana & 0 & 20 & 0 & 20 & 4 \\
\hline Campanella alba & 17 & 48 & 30 & 95 & 21 \\
\hline Coprinus disseminatus & 2 & 0 & 8 & 10 & 2 \\
\hline Marasmius cladophyllus & 0 & 5 & 6 & 11 & 2 \\
\hline Marasmius quercophilus & 0 & 15 & 22 & 37 & 8 \\
\hline Mycena renati & 8 & 25 & 22 & 55 & 12 \\
\hline Pluteus nanus & 10 & 2 & 10 & 22 & 5 \\
\hline Pluteus salicinus & 10 & 2 & 10 & 22 & 5 \\
\hline Xilarya polymorpha & 5 & 30 & 2 & 37 & 8 \\
\hline
\end{tabular}

Para el análisis de la diversidad de especies se utilizó el paquete Vegan de R versión 3.1.2. Con éste se calculó el índice de diversidad de Shannon y su inverso para conocer cuáles de los sitios muestreados fueron los más diversos para los años 2013 y 2014. A este índice se le aplicó la función del Invs para incrementar los valores obtenidos con el índice de Shannon y así lograr una mejor interpretación de los datos.

En relación al índice de Shannon y su inverso Inv., se encontró que los sitios o parcelas más diversos fueron aquellos que se ubicaron en el bosque tipificado como Roble con valores que oscilan entre (2.26 y 2.85) para el año 2013 y (2 y 2.70) para el año 2014. Cabe señalar que con este índice los sitios con un grado de significancia en términos de diversidad son interpretados a partir del valor numérico 3, por lo que en este análisis se están tomando en cuenta los valores del índice más cercanos al número 3.

Las parcelas ubicadas en los sitios denominados como Tacotal fueron las que presentaron valores inferiores a 2 al aplicar el índice de Shannon. Estas parcelas presentaron valores de (1.83 a 1.98) durante el año 2013, situación que cambio de forma negativa durante el muestreo de 2014 ya que mostraron valores muy por debajo de 0.7. Durante el muestreo de octubre de 2014, el sitio que muestra el índice de Shannon más cercano al biotopo caracterizado como Roble es el biotopo denominado Ripario, con valores que oscilan entre 1 y 1.70. 
a

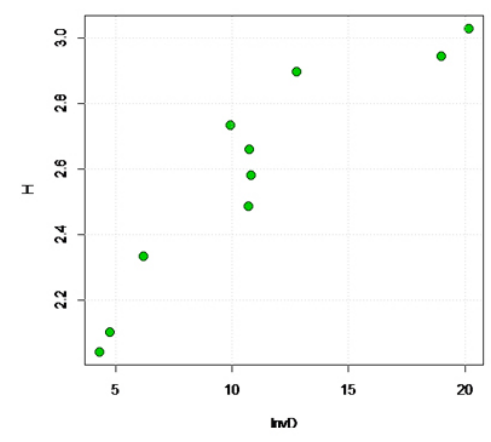

b

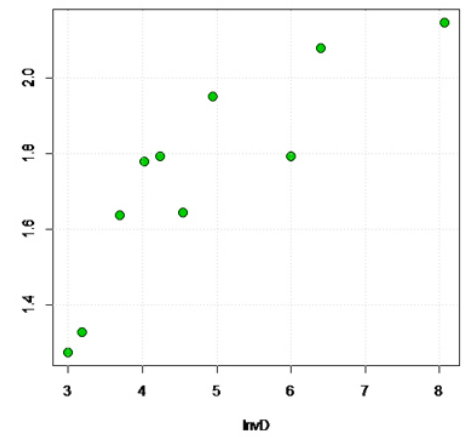

Ilustración 3. Izq. Índice de diversidad de especies de Shannon e Inv.: a) año 2013 y b) año 2014

En la ilustración 3 se observa que la tendencia de la curva de especies aún no se estabiliza, siendo posible encontrar todavía especies nuevas en el área. Para mejorar la interpretación de este gráfico se realizó una prueba de rarefacción con el objetivo de conocer si la cantidad de especies encontradas en el último muestreo alcanzó su asíntota máxima. Se interpretó aquí, como resultado, que la asíntota de nuestro gráfico puede seguir creciendo, lo cual nos indicaría que el número de especies de macrohongos en la zona podría aumentar (iustración 4).
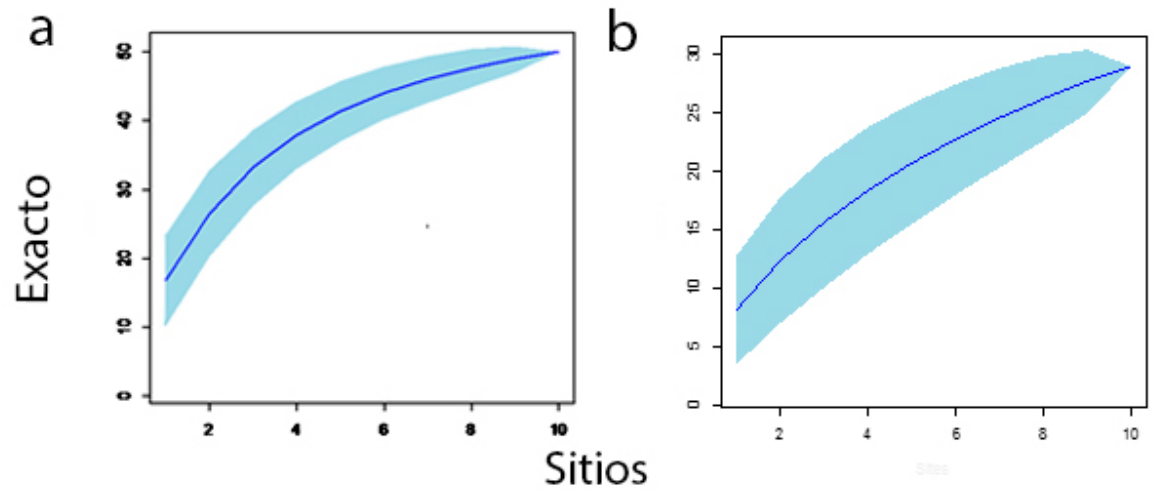

Ilustración 4. Prueba de rarefacción utilizando los valores de los inversos del índice de Shannon datos 2013 (a) en función del número de especies encontradas y los sitios muestreados

Se ejecutó un test de similaridad basado en datos de presencia ausencia, para entender el comportamiento de las especies en los puntos muestreados. Para ello se utilizó el paquete Vegan de R-Project, en donde se llamó a las funciones vegdist (average) y hclus. Estas funciones permiten calcular la distancia promedio de especies en los sitios muestreados. De los diferentes dendrogramas obtenidos se eligió el dendrograma "average", el cual se ajusta de manera más clara a la interpretación de los datos. 
El resultado para el muestreo de 2013 fue de tres grupos bien definidos de comunidades de macrohongos en las parcelas ubicadas en los parches de bosque de la Estación Biológica Juan Roberto Zarruck. Los sitios 1, 2, 6, 7, 8, 9 representan la zona denominada bosque de roble y tienen un nivel máximo de similaridad de 0.70 , y en el caso de los sitios 1 y 2 (0.50) éstos representan el sitio denominado Tacotal. Los sitios 4, 3, 5 están asociados en una escala 0.40 con respecto al resto de los grupos y pertenecen al sitio denominado como bosque ripario.

a

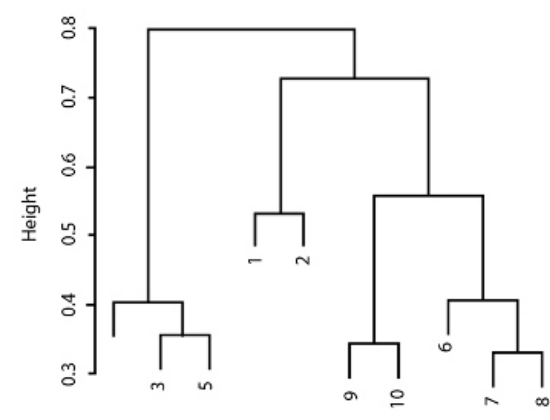

$\mathrm{b}$

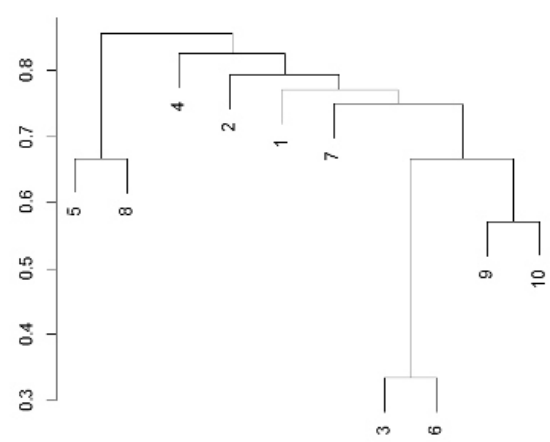

Ilustración 5. Índices de similitud de Jaccard de izquierda a derecha años 2013 y 2014

Como resultado del muestreo de 2014 fueron encontrados dos grupos de comunidades de macrohongos que para el año 2013 no fueron tan definidas. Esto puede deberse al traslape de especies en la distribución espacial. La similaridad entre sitios resultó de 0.84 y en el caso de los sitios 3 y 6 fueron los únicos en mostrar un valor inferior (0.4). Este análisis incluso nos muestra la tendencia de que sólo existe una comunidad debido a la similaridad observada entre los sitios con un valor superior a 0.50. Ello indica que existe una comunidad homogénea.

Se realizó un análisis de diversidad según el número de parcelas correspondientes a cada biotopo muestreado, por lo que se encontraron los siguientes valores para el índice de Shannon, según biotopos:

Cuadro 5. Valores Índice de diversidad de Shannon de colectas en función del tipo de biotopo

\begin{tabular}{|c|c|c|c|}
\hline & Tacotal & Ripario & Roble \\
\hline 2013 & 1,921569 & 2,218643 & 2,573301 \\
\hline 2014 & 1,623743 & 1,876343 & 1.921 .569 \\
\hline
\end{tabular}

Índice de diversidad de Shannon ideal $<3$.

Los resultados del clúster de Jaccard y los resultados del índice de diversidad los podemos confirmar al observar el cuadro 6. Éste muestra la presencia y ausencia de especies según nuestras caracterizaciones de biotopo. 
Cuadro 6. Conteos de especies según el tipo de bosque y el sustrato - año 2013

\begin{tabular}{|c|c|c|c|c|c|c|c|}
\hline & Especies & Tacotal & BosRob & Ripario & Terrestre & Mad.Sap & Hoja \\
\hline 1 & Amanita brunnescens & & $\mathrm{B}$ & & $\mathrm{T}$ & & \\
\hline 2 & Auricularia fuscosuccinea & & $\mathrm{B}$ & $\mathrm{R}$ & & & \\
\hline 3 & Bierkandera adusta & Tac & & $\mathrm{R}$ & & $\mathrm{M}$ & \\
\hline 4 & Camillea sagrana & & $\mathrm{B}$ & & & $\mathrm{M}$ & \\
\hline 5 & Campanella alba & Tac & $\mathrm{B}$ & $\mathrm{R}$ & & $\mathrm{M}$ & \\
\hline 6 & Chlorociboria sp & Tac & $\mathrm{B}$ & $\mathrm{R}$ & & $\mathrm{M}$ & \\
\hline 7 & Collybia aurea & Tac & $\mathrm{B}$ & & & $\mathrm{M}$ & \\
\hline 8 & Cookeina speciosa & Tac & $\mathrm{B}$ & $\mathrm{R}$ & & $\mathrm{M}$ & \\
\hline 9 & Coprinus disseminatus & Tac & $\mathrm{B}$ & & $\mathrm{T}$ & $\mathrm{M}$ & \\
\hline 10 & Coprinus sp & $\mathrm{Tac}$ & & & & $\mathrm{M}$ & \\
\hline 11 & Crinipellis procera & Tac & $\mathrm{B}$ & $\mathrm{R}$ & & $\mathrm{M}$ & $\mathrm{H}$ \\
\hline 12 & Crinipellis scabella & Tac & & $\mathrm{R}$ & & $\mathrm{M}$ & $\mathrm{H}$ \\
\hline 13 & Crinipellis stipitaria & Tac & $B$ & $\mathrm{R}$ & & $\mathrm{M}$ & $\mathrm{H}$ \\
\hline 14 & Cyathus striatus & Tac & $\mathrm{B}$ & & $\mathrm{T}$ & $\mathrm{M}$ & \\
\hline 15 & Cyclomyces tabacinus & Tac & $\mathrm{B}$ & & & $\mathrm{M}$ & \\
\hline 16 & Cystolepiota sistrata var. sistrata & Tac & $\mathrm{B}$ & & & $\mathrm{M}$ & \\
\hline 17 & Dacryopinax spathularia & Tac & B & & & $\mathrm{M}$ & \\
\hline 18 & Dictyopanus pusillus & $\mathrm{Tac}$ & & & & $\mathrm{M}$ & \\
\hline 19 & Entoloma sp & & $\mathrm{B}$ & & $\mathrm{T}$ & & \\
\hline 20 & Filoboletus gracilis & & $\mathrm{B}$ & & & $\mathrm{M}$ & \\
\hline 21 & Geastrum saccatum & Tac & $\mathrm{B}$ & & $\mathrm{T}$ & & \\
\hline 22 & Gymnopilus sp & & & $\mathrm{R}$ & & $\mathrm{M}$ & \\
\hline 23 & Helvella atra & & $\mathrm{B}$ & & $\mathrm{T}$ & & \\
\hline 24 & Hohenbuelia geogenia & Tac & $\mathrm{B}$ & & & $\mathrm{M}$ & \\
\hline 25 & Hypholoma subviride & Tac & $\mathrm{B}$ & & $\mathrm{T}$ & $\mathrm{M}$ & \\
\hline 26 & Leotia lubrica & & $\mathrm{B}$ & & $\mathrm{T}$ & & \\
\hline 27 & Leucocoprinus fragilissimus & Tac & $\mathrm{B}$ & & $\mathrm{T}$ & & \\
\hline 28 & Marasmius aripoensis & Tac & $\mathrm{B}$ & & & & \\
\hline 29 & Marasmius cladophyllus & Tac & $\mathrm{B}$ & & & $\mathrm{M}$ & \\
\hline 30 & Marasmius haematocephalus & & $\mathrm{B}$ & & & $\mathrm{M}$ & \\
\hline 31 & Marasmius helvolus & & $\mathrm{B}$ & $\mathrm{R}$ & & $\mathrm{M}$ & \\
\hline 32 & Marasmius leprieurii & Tac & B & & & $\mathrm{M}$ & $\mathrm{H}$ \\
\hline 33 & Marasmius sp & Tac & $\mathrm{B}$ & & & $\mathrm{M}$ & $\mathrm{H}$ \\
\hline 34 & Marasmius perlongispermus & & B & & & M & \\
\hline 35 & Marasmiellus volvatus & Tac & $\mathrm{B}$ & $\mathrm{R}$ & & $\mathrm{M}$ & \\
\hline 36 & Mycena margarita & Tac & & & & $\mathrm{M}$ & \\
\hline 37 & Mycena pallida & Tac & $\mathrm{B}$ & & $\mathrm{T}$ & $\mathrm{M}$ & \\
\hline 38 & Mycena sanguinolenta & Tac & $\mathrm{B}$ & $\mathrm{R}$ & $\mathrm{T}$ & $\mathrm{M}$ & \\
\hline 39 & Naucoria striatula & & $\mathrm{B}$ & & & $\mathrm{M}$ & \\
\hline 40 & Phellinus giluus & & $B$ & & & $M$ & \\
\hline 41 & Phillipsia domingensis & & $\mathrm{B}$ & & & $\mathrm{M}$ & $\mathrm{H}$ \\
\hline 42 & Pluteus leoninus & Tac & & & & $\mathrm{M}$ & $\mathrm{H}$ \\
\hline 43 & Pluteus salicinus & Tac & $\mathrm{B}$ & & & $\mathrm{M}$ & \\
\hline 44 & Podostroma alutaceum & $\mathrm{Tac}$ & & & $\mathrm{T}$ & $\mathrm{M}$ & \\
\hline
\end{tabular}




\begin{tabular}{|l|l|c|c|c|c|c|c|}
\hline 45 & Ramaria stricta & & $\mathrm{B}$ & $\mathrm{R}$ & $\mathrm{T}$ & & \\
\hline 46 & Ramaria zippelii & & $\mathrm{B}$ & & $\mathrm{T}$ & & \\
\hline 47 & Scutellinia scutellata & $\mathrm{Tac}$ & $\mathrm{B}$ & $\mathrm{R}$ & & $\mathrm{M}$ & \\
\hline 48 & Tetrapyrgos nigripes & & $\mathrm{B}$ & $\mathrm{R}$ & & $\mathrm{M}$ & \\
\hline 49 & Trametes versicolor & $\mathrm{Tac}$ & $\mathrm{B}$ & & & $\mathrm{M}$ & \\
\hline 50 & Tricholoma myomyces & $\mathrm{Tac}$ & & & & $\mathrm{M}$ & \\
\hline 51 & Xylaria hypoxylon & $\mathrm{Tac}$ & $\mathrm{B}$ & $\mathrm{R}$ & $\mathrm{T}$ & $\mathrm{M}$ & \\
\hline 52 & Xylaria polymorpha & $\mathrm{Tac}$ & $\mathrm{B}$ & $\mathrm{R}$ & & $\mathrm{M}$ & \\
\hline & Totales & 35 & 43 & 17 & 15 & 42 & 8 \\
\hline
\end{tabular}

En la misma tabla es posible visualizar la preferencia de los sustratos de las especies muestreadas en el año 2013. De una muestra total de 52 especies, se encontraron 42 especies de hábitos saprofitos de madera en descomposición. De estas 42 especies saprofitas, 28 especies fueron encontradas exclusivamente en el sustrato madera. El segundo sustrato importante, resulto ser tierra, con once especies. Los macrohongos encontrados sobre hojarasca fueron ocho.

La selección de sustrato de los macrohongos también varía en algunas especies: así encontramos al menos seis especies que pueden encontrarse tanto sobre madera, como sobre tierra. Estas especies son: Hypholoma subviride, Mycena sanguinolenta, Podostroma alutaceum, Xylaria hypoxylon, Cyathus striatus, Coprinus disseminatus. También se identificó que las especies Amanita brunnescens, Entoloma sp, Ramaria stricta y Ramaria zippelii fueron especies que se encontraron exclusivamente sobre tierra.

El muestreo realizado en octubre de 2014 arrojó los siguientes datos de distribución y preferencia de sustrato:

Cuadro 7. Conteos de especies según el tipo de bosque y el sustrato año 2014

\begin{tabular}{|l|l|l|l|l|l|l|l|}
\hline & Especies & Tacotal & BosRob & Ripario & Terrestre & Madera & Hoja \\
\hline 1 & Auricularia auricularia-judae & Tac & & & & M & \\
\hline 2 & Bjerkandera adusta & Tac & & & & M & \\
\hline 3 & Calvatia rugosa & & R & & & M & \\
\hline 4 & Camillea sagrana & Tac & R & Rip & & M & \\
\hline 5 & Campanella alba & Tac & R & Rip & & M & \\
\hline 6 & Collybia neotropica & Tac & & Rip & & M & \\
\hline 7 & Coprinus disseminates & Tac & & & T & M & \\
\hline 8 & Crinipellis procera & Tac & R & & T & M & \\
\hline 9 & Crinipellis scabella & & & & T & M & \\
\hline 10 & Crinipellis stipitaria & Tac & & & & M & \\
\hline 11 & Daedalea quercina & & Rob & Rip & & M & \\
\hline 12 & Datronia caperata & & Rob & Rip & & M & \\
\hline 13 & Dictyopanus pusillus & Tac & & & & M & \\
\hline
\end{tabular}




\begin{tabular}{|c|c|c|c|c|c|c|c|}
\hline 14 & \multicolumn{2}{|l|}{ Earliella scabrosa } & Rob & & & M & \\
\hline 15 & \multicolumn{2}{|l|}{ Engleromyces goetzei } & Rob & & $\mathrm{T}$ & $\mathrm{M}$ & \\
\hline 16 & \multicolumn{2}{|l|}{ Gymnopus iocephalus } & Rob & & & M & \\
\hline 17 & \multicolumn{2}{|l|}{ Gymnopus erythropus } & Rob & & $\mathrm{T}$ & M & \\
\hline 18 & Lepiota subcristata & Tac & Rob & & $\mathrm{T}$ & $M$ & \\
\hline 19 & \multicolumn{2}{|l|}{ Leucocoprinus cepistipis } & Rob & & $\mathrm{T}$ & M & \\
\hline 20 & \multicolumn{2}{|l|}{ Leucocoprinus fragilissimus } & Rob & Rip & $\mathrm{T}$ & $\mathrm{M}$ & \\
\hline 21 & \multicolumn{2}{|l|}{ Marasmius berteroi } & Rob & & & M & \\
\hline 22 & Marasmius cladophyllus & Tac & Rob & & $\mathrm{T}$ & $\mathrm{M}$ & $\mathrm{H}$ \\
\hline 23 & \multicolumn{2}{|l|}{ Marasmiellus guadelupensis } & Rob & & $\mathrm{T}$ & $\mathrm{M}$ & $\mathrm{H}$ \\
\hline 24 & \multicolumn{2}{|c|}{ Marasmius haematocephalus } & Rob & & & $\mathrm{M}$ & $\mathrm{H}$ \\
\hline 25 & Marasmius quercophyllus & Tac & Rob & Rip & & $\mathrm{M}$ & $\mathrm{H}$ \\
\hline 26 & \multicolumn{2}{|l|}{ Marasmius tageticolor } & Rob & & & $\mathrm{M}$ & $\mathrm{H}$ \\
\hline 27 & \multicolumn{2}{|l|}{ Mycena chusqueophila } & Rob & Rip & & $M$ & $\mathrm{H}$ \\
\hline 28 & Mycena pallida & Tac & Rob & Rip & & $\mathrm{M}$ & $\mathrm{H}$ \\
\hline 29 & Mycena renati & Tac & Rob & Rip & & $M$ & \\
\hline 30 & Pluteus nanus & Tac & Rob & Rip & $\mathrm{T}$ & $M$ & \\
\hline 31 & \multicolumn{2}{|l|}{ Pluteus salicinus } & Rob & & & $M$ & \\
\hline 32 & \multicolumn{2}{|l|}{ Psathyrella conopilus } & Rob & & & $\mathrm{M}$ & \\
\hline 33 & \multicolumn{2}{|l|}{ Tremella mesenterica } & Rob & & & $\mathrm{M}$ & \\
\hline 34 & Xylaria hypoxylon & Tac & Rob & & & $\mathrm{M}$ & \\
\hline 35 & \multicolumn{2}{|l|}{ Xylaria polymorpha } & Rob & & $\mathrm{T}$ & $\mathrm{M}$ & \\
\hline & Total & & & & 12 & 35 & 7 \\
\hline
\end{tabular}

La tabla anterior nos muestra los resultados del muestreo de datos correspondiente al año 2014. Se encontraron 35 especies saprofitas de madera en descomposición, lo cual representa el 100\% de las especies de este muestreo. Doce especies se encontraron sobre el sustrato terrestre o micelios establecidos en tierra y sólo siete especies utilizaron hojas como sustrato.

La selección del sustrato varía en algunas especies. Se reportan dos especies adaptables a los tres sustratos aquí analizados: Marasmius cladophylus y Marasmius guadelupensis.

\section{Discusión}

El presente estudió identificó la presencia de 73 especies de macrohongos, siendo la taxa predominante del orden Agaricales. Se encontraron 33 nuevos reportes para la Estación Biológica Juan Roberto Zarruck, a partir de la creación del listado de especies en el año 2012. Los macrohongos son uno de los taxones en Nicaragua y Centroamérica cuyo número de especies se encuentra en continuo aumento. Este aumento del número de especies está asociado al aumento de los esfuerzos 
muestrales a diferentes escalas y con diferentes objetivos (Rosenzweig, 1996).

En la Finca Santa Maura la diversidad de hongos encontrados durante el primer esfuerzo de muestreo en las parcelas permanentes no fue satisfactorio. Todos los sitios mostraron una diversidad por debajo de tres, que en el caso del índice de Shannon sería el valor óptimo con el que se empiezan a detectar sitios diversos. Sin embargo, según datos obtenidos al realizar más esfuerzos muestrales podemos seguir encontrando especies, ya que la curva de diversidad de especie aún no se estabiliza (ilustración 4), sino que sigue una proyección ascendente.

Los valores de Shannon para el muestreo realizado en el 2013 fueron de 2.85, un valor que para este índice se interpreta como poco diverso, y en el año 2014 este valor incluso descendió a 2.70. La reducción de los valores de diversidad para el año 2014 podría estar influenciada por una leve disminución de las precipitaciones debido a la sequía ocurrida en Nicaragua en ese año. Esto podría responder a que la aparición de hongos depende de muchos factores bióticos y abióticos que ocurren a nivel de microhábitat (Rodríguez, Cullen, Kurtzman, Kachatourians \& Hegedus, 2004). Por esto cambios leves en las condiciones de humedad pueden variar el comportamiento de estos organismos en relación a su microhábitat.

Estudios hechos durante una década de colectas en parcelas permanentes han dado indicios que existe una relación entre la variación en la fructificación de los hongos y variaciones en los parámetros ambientales asociados a cambios en las precipitaciones (Kauserud et al., 2008). Investigaciones de distribución en espacio y tiempo han demostrado que la disminución en un 30\% de la precipitación puede disminuir hasta en un 60\% la fructificación de hongos (Egli, 2011).

Estudios específicos de especies de macrohongos han indicado que especies como Auricularia judae experimentan variaciones importantes de fructificación y de selección de sustrato ligadas al cambio climático (Gange, Gange, Aquila \& Boddy, 2011). Además, las variaciones en la fructificación de hongos han demostrado tener consecuencias directas en los procesos de reciclaje del bosque (Gómez, Gallegos, Albores \& Sánchez, 2007).

A pesar de los valores indicados por el índice de Shannon en nuestro estudio, sí hubo aproximaciones importantes al ideal de diversidad. Éstas fueron encontradas en el biotopo denominado bosque de roble, que mostró valores muy cercanos a tres según el índice de Shannon en ambos años de estudio. El bosque de roble fue denominado así a causa de la marcada presencia de roble encino, así como de muchas especies de melastomatácea, combretácea y epifitas. Todas estas especies representan taxones que tienen algún nivel de dependencia de patrones de humedad e incluso, en algunos casos, de los parámetros altitudinales (Stevens, 1995). Estos también son parámetros importantes para definir la presencia o ausencia de hongos en un hábitat (Hawksworth, 1989; Rodríguez et al., 2004).

Otros estudios demuestran que los robledales están asociados a comunidades fúngicas concretas, entre las que destacan muchas ectomicorrizas y basidiomicetes (Ortega Martínez \& Martínez Peña, 2008). En los resultados de este estudio, a pesar de la baja presencia de hongos ectomicorrízicos, se encontraron especies específicamente asociadas a robledales, tales como: Helvella atra, Marasmius, Collibya, Geastrum saccatum, Calvatia rugosa, Marasmius quercophilus (Gómez et al., 2007) y Amanita brunnences (Mata, 2003). También muchas de las especies encontradas, 
como en el caso de los Xylariales que son en principio hongos pioneros (Gange et al., 2011), podrían estar indicando una primera etapa en el reciclaje en las parcelas analizadas en la Estación Biológica Juan Roberto Zarruck.

En el caso de los hábitats denominados como tacotal y ripario, éstos son parches de bosque en los cuales se puede percibir un mayor nivel de perturbación que puede ser fácilmente percibido en los estratos de la composición vertical de la vegetación (Dierschke, 1994) e indica la presencia de hierbas y arbustos pequeños. Sin embargo, el análisis específico del índice de Shannon aplicado a los biotopos por individual mostró valores entre 2.7 y 1.9 para los años 2013 y 2014. Ello nos indica que en esta zona podrían estar ocurriendo recambios de especies posiblemente asociados al efecto del río como zona de transición y transporte entre biotopos.

Las especies encontradas de manera recurrente en los biotopos denominados tacotal y ripario fueron especies asociadas a la indicación de perturbaciones ambientales. Éstas además pueden ser reforzadas por una marcada presencia de lianas y de vegetación asociada a estados de regeneración del bosque. En cuanto a la composición de la comunidad fúngica encontrada en esta área se puede inferir que se encontraron especies típicas para este tipo de hábitat perturbado, sobre todo por la presencia de las siguientes especies: Marasmius (García \& Bolaños, 2010), Cyathus striatus, Xylaria, Phellinus y Polyporus, Camillea, Scutellinia, Ramaria, Engloromyces goetzei (Gómez et al., 2007). En el caso de Xylaria es un género que se considera pionero, por lo tanto es una especie tolerante a la perturbación de hábitat.

Las relaciones en la proporción de la riqueza y abundancia de especies encontradas pueden estar influidas por diferentes factores, como la época de fructificación que normalmente ocurre de julio a octubre (Mata, 2003). Sin embargo puede estar siendo afectada por variaciones climáticas que repercuten en la fructificación. Estudios recientes han puesto en evidencia que las variaciones de la precipitación en términos espacio temporal pueden repercutir directamente en alargar o atrasar los períodos de fructificación de algunas especies de hongos, siendo los meses más sensibles agosto y octubre (Kauserud et al., 2008).

Por otra parte, algunas de las parcelas tenían una pendiente de inclinación pronunciada, por lo que algunos sitios en donde se ubicaron las parcelas pueden estar expuestos a factores de erosión de tipo dinámica a causa de la escorrentía. En este aspecto lo ideal sería poder analizar especies frecuentes en relación a su sustrato o especies arbustivas asociadas y revisar si existe una ausencia absoluta del organismo o si al menos éstos se encuentran en estado micelio en los sustratos (Gange et al., 2011).

Los niveles de diversidad de especies y sus abundancias en los hongos responden a niveles más complejos de agrupación que en el caso de las plantas. Esto se debe a que para los hongos fuera de las influencias del entorno ambiental existen además requerimientos muy específicos para los que aún no hay patrones estandarizados. Así, por ejemplo, el microhábitat juega en muchas ocasiones un rol más fuerte que el hábitat, debido a que las especies están fuertemente determinadas por el sustrato, cambios de humedad, de $\mathrm{pH}$ e incluso por sus funciones bioquímicas como respuesta al medio (Rodríguez et al., 2004; Müller, Bills \& Foster, 2004).

Debido a todos estos factores ambientales y de relaciones intraespecíficas de los hongos es que el sustrato puede llegar a tener un valor decisivo en la interpretación 
de su dinámica dentro del ecosistema. En este estudio encontramos que el $80 \%$ de las especies (42) fueron saprofitas de madera en descomposición en el año 2013. Además, el 100\% resultaron saprofitas lignícolas en el 2014. Muy raramente se encontraron especies humícolas, sobre suelo u hojarasca. La abundancia de los hongos saprofitos y no parásitos muestran y reafirman la gran labor de reciclaje de materia muerta que realizan estas especies en el ecosistema, lo cual es uno de los mayores atributos de los hongos (Müller et al., 2004).

Los hongos son colaboradores del reciclaje. De ahí que los hongos con un diámetro de micelio de 2 a 10 micras contenidos en un litro de material o sustrato adecuado son capaces de reciclar una hectárea de material lignícola en un bosque (Osono, 2007). Por ello, su labor recicladora resulta invaluable. Además significa que es muy difícil entender todos los posibles efectos colaterales resultantes de una disminución dramática de estos organismos en el bosque. Muchas especies están intrínsecamente asociadas a determinados arboles, así que la producción de micorrizas es proporcional a la producción de carbohidratos de ciertas especies de árboles y viceversa (Egli, 2011).

El análisis del comportamiento de la composición y distribución de la flora fúngica en los parches de bosque de la Estación Biológica Juan Roberto Zarrück nos muestra que existe un desbalance. Asimismo podría estar ocurriendo una posible pérdida de diversidad en hongos en esta reserva. Ello tiene que ser observado con detenimiento debido a las implicaciones ecológicas de dicho fenómeno. Para poder lograr robustez en los datos es necesario prolongar el tiempo de observación de las parcelas en estudio. Sin embargo, los resultados obtenidos no dejan de ser importantes. Éstos nos alertan de una posible tendencia al descenso de la riqueza de especies fúngicas de la zona, lo que podría afectar aspectos importantes del reciclaje de nutrientes del bosque, siempre y cuando esto no fuese un fenómeno aislado en el año 2014.

Otro factor a tomar en cuenta en muestreos posteriores es el seguimiento de la masa miceliar de estos organismos en relación a los sustratos en los que se encuentran. Estos datos no sólo contribuirían a establecer de forma robusta el estado en que se encuentran estos organismos si no que facilitarían datos específicos de la cantidad de material que están reciclando de forma anual. Ello permite establecer cuotas de pérdida o ganancia en el material reciclado a largo plazo.

\section{Conclusiones}

En los años 2013 y 2014 se llevaron a cabo muestreos fúngicos en parcelas permanentes ubicados en parches de bosque localizados en la Finca Santa Maura, Estación Biológica Roberto Zarruck. Durante el estudio se encontró una riqueza de 73 especies de macrohongos.

Se recolectaron 14 especies nuevas para el listado de especies de Nicaragua. La composición de especies en cuanto a su riqueza y abundancia mostró variaciones en el año 2013 con respecto al 2014, siendo la riqueza de 52 especies reportadas en el 2013 y 35 especies en el 2014. También varió la abundancia de especies de 1068 en el 2013 a 448 en el 2014. En ambos años el índice de riqueza de Shannon no mostró 
un valor óptimo $(<3)$ de riqueza de especies.

El 57 \% (2013) y el 47\% (2014) de las especies encontradas son de hábitos saprofitos, lo que indica que las especies encontradas son principalmente recicladoras de materia orgánica.

Los resultados obtenidos nos indican que hay que seguir investigando para poder establecer si la variación en el número y abundancia de especies se debió a un fenómeno aislado (precipitaciones en 2014) o es una posible tendencia que indica variaciones de la composición fúngica producto de alteraciones en la zona asociadas a las precipitaciones de la zona.

\section{Agradecimientos}

Este trabajo fue posible gracias a la amable cooperación del CIDEA de la UCA en la fase de laboratorio. La autora agradece específicamente la ayuda en campo y revisión del manuscrito a Marvin Tórrez, coordinador de la Estación Biológica Juan Roberto Zarruck. La Estación Biológica agradece a Paso Pacífico y al programa de monitoreo que se realiza en conjunto con el USDA-FS, cuyas parcelas permanentes sirven de referencia espacial para este muestreo. Es especial el agradecimiento a la familia Chávez y en especial a Don Jorge Chávez por su apoyo a la conservación de Nicaragua. Por su aporte en la revisión en inglés del resumen se agradece a Miguel Garmendiaz.

\section{Referencias bibliográficas}

Breuss, O. (2003). Flechten aus Nicaragua. Recuperado el 05 de octubre de 2014, de: http://www.landesmuseum.at/pdf_frei_remote/LBB_0034_2_1053-1069. pdf

Cesefor: Gestión Forestal Sostenible. (2013). Hongos Como Indicadores de Cambio Climático. Recuperado el 05 de octubre de 2014, de: http://www.dicyt.com/ noticias/cesefor-investiga-efectos-del-cambio-climatico-en-las-setas-y-en-elcrecimiento-de-arboles.

Delgado, G. (2011). Nicaraguan Fungi: A Checklist of Hyphomycetes. Mycotaxon, (115), 534.

Dierschke, H. (1994). Pflanzensoziologie. Sttutgart: Eugen Ulmer Verlag.

Egli, S. (2011). Mycorrhizal Mushroom Diversity and Productivity: An Indicator of Forest Health?. Annals of Forest Science, (68), 81-88.

Gange, A. C, Gange, E. G, Aqilah, M. B, \& Boddy, L. (2011). Host Shifts in Fungi Caused by Climate Change?. Fungal Ecology, (4), 184-190.

García, L., A. \& Bolaños, R. A.C. (2010). Macrohongos Presentes en el Bosque Seco Tropical de la Región del Valle del Cauca, Colombia. Revista de Ciencias Universidad del Valle, 14 (86), 45-54.

González, I. \& Orozco, T. (2007). Inventario de Macrohongos Reserva Natural Indio Maíz. Documento inédito. Managua: Araucaria Río San Juan-Ministerio de Ambiente y Recursos Naturales.

Gómez, F. C., Gallegos, A.H.R., Albores, J.C., Sánchez, J.E. (2007). Macromicetos 
del Parque Educativo Laguna Bélgica, Municipalidad de Ocozocoautla de Espinosa, Chiapas México. Revista Mexicana de Biodiversidad, 7, 369-381.

Hawksworth, D. (1989). Interacciones Hongo, Hongo-Alga en Simbiosis Liquénicas y Liquenoides. Anales Jardín Botánico de Madrid, 46 (1), 235-247.

Kauserud, H., Stige, L.C., Vik, J.O., Økland, R.H., Høiland, K. \& Stenseht, N.C.

(2008). Muschroom Fruiting and Climate Change. PNAS, 105 (10), 38113814.

Mata, M. (2003). Macrohongos de Costa Rica. (Vol.1). Costa Rica: Inbio.

Müller, E. \& Loefller, W., (1976). Micología: Manual para Naturalistas y Médicos. Barcelona: Omega.

MushroomExpert. (2015). MushroomExpert: Claves de Xylaria. Recuperado el 02 de enero de 2015, de: http://www.mushroomexpert.com/xylaria.

Müller, G., Bills, G. \& Fosters, M. (2004). Mushrooms Monitoring Species. Biodiversity of Fungies: Inventories and Methods. Preface. Elsevier, 2, 2-4.

Ministerio de Medioambiente Recursos Naturales [MARENA] \& Fundación Amigos del Río San Juan [FUNDAR]. (2012): Actualización de Plan de Manejo: Reserva Natural Cerro Datanli-El Diablo. Managua: FUNDAR.

Ortega Martínez, P. \& Martínez Peña, F. (2008). A Sampling Method for Estimating Sporocarps Production of Wild Edible Mushrooms of Social and Economic Interest. Investigación Agraria: Sistemas y Recursos Forestales, 17(3), 228-237.

Osono, T. (2007). Ecology of Ligninolytic Fungi Associated with Leaf Litter Descomposition. Ecology Reviews, (22), 955-974.

Pegler, D.N. (1983). Agaric Flora of the Lesser Antilles. Kew Bulletin, Additionals Series IX. Kew: London Royal Botanicals Gardens.

Petersen, J. H. \& Læssøe, T. (2014). Mycokey: The Mycological Information Site. Claves de Macrohongos. Recuperado el 01 de noviembre de 2014 de: http://www. mycokey.org/result_textsearch.shtml?genSpec $=$ Species\& $\& D=1436 \&$ showList $=$ DropDown\& random $=18770$

Rosenzweig, M. L. (1996). Species Diversity in Space and Time. New York: Cambridge University Press.

Rodríguez, J. R., Cullen, D., Kurtzman, C.P., Kachatourians, G.G. \& Hegedus, D.D. (2004). Molecular methods for discriminating taxa, monitoring species, and assessing fungal diversity. Biodiversity of fungies: Inventories and Methods. Elsevier. (2).79.

Shannon, C. E. \& Weaver, W. (1948). A Mathematical Theory of Communication. Bell System Technical Journal, 27 (3), 379-423.

Stevens, W. (1995). Bosque de Robles: Anales de la Flora de Nicaragua. Recuperado el 01 de noviembre de 2014, de: http://www.mobot.org/MOBOT/research/ nicaragua/flora.shtml 